\title{
Electrochemical Characterization of Poly(acrylamide) Membrane Coated on a Gold Disk Electrode in Acetonitrile
}

\author{
Xueping Ji ${ }^{\mathrm{a}}$, Baokang Jin ${ }^{\mathrm{a}, \mathrm{b}}$, Jujie Ren ${ }^{\mathrm{a}}$, Jiye Jin ${ }^{\mathrm{a}}$, and Toshio Nakamura ${ }^{\mathrm{a} *}$

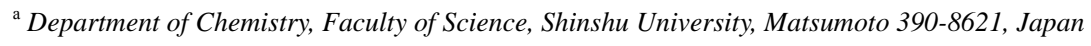 \\ ${ }^{\mathrm{b}}$ Department of Chemistry, Anhui University, Hefei, Anhui 230039, PR China
}

* Corresponding author. Tel. \& fax: + 81-37-2507.

E-mail address: toshion@gipac.shinshu-u.ac.jp

Published in: Journal of Electroanalytical Chemistry. 579(1): 25-31 (2005) 


\begin{abstract}
The electrochemical behavior of a poly(acrylamide) (PAA) membrane electrode (ME), in which the PAA works as a membrane matrix for use in nonaqueous solutions, was investigated in acetonitrile. It was found that PAA MEs possess properties of microelectrode ensembles (MEEs). For the thin PAA membrane, the electrode behaved similar to a macroelectrode and peak-shaped voltammograms were achieved at low scan rates, while at high scan rates the electrode acted as a microelectrode and sigmoidal-shaped voltammograms were obtained. For the thick PAA membrane electrode, however, voltammograms were sigmoidal-shaped at low scan rates, and at high scan rates the voltammograms became peak-shaped again. Quantitative and semiquantitative analyses of the electrochemical response characteristics of these ensembles were conducted. The results show that the electrochemical behavior of these ensembles is in close agreement with the predictions of established microwell electrochemical theory.
\end{abstract}

Keywords: Microelectrode ensembles; Poly(acrylamide); Ferrocene; Acetonitrile 


\section{Introduction}

Polymer membrane ion-selective electrodes are very useful for detecting the activities of ions in solution due to their good reproducibility, stability, and simplicity. Many of these polymer electrodes have been discussed from the viewpoint of potential response mechanism, lower detection limit, and selectivity coefficient [1-4]. Polymer membranes have also been used as a matrix in enzyme electrode [5-7]. But in the majority of cases, they have been used in water solution.

A number of cation [8,9] and anion [10] selective electrodes and biosensors [11] were developed at our laboratory using a non-plasticized hydrophilic poly(acrylamide) (PAA) as membrane matrix for use in dipolar aprotic solvents and in the study of the thermodynamic properties of ions. Studies of the response mechanisms of the electrodes were also carried out by voltammetry and spectrophotometry $[12,13]$. We supposed that the PAA membrane possessed porous properties, and that gold and platinum electrodes modified with PAA membrane might yield microelectrode ensembles (MEEs) when the membrane was not very thick. It is important to understand the electrochemical properties of PAA as sensing membrane matrix.

We report here the electrochemical behavior of PAA membrane in nonaqueous solutions investigated by voltammetric and chronoamperometric experiments in order to characterize the function of the membrane electrode. Quantitative and semiquantitative analyses of the electrochemical response characteristics of these membrane electrodes (ME) were also conducted in this study. The results agree well both with the predictions of established microwell electrochemical theory and with the results of previous investigations $[14,15]$.

\section{Experimental}

\subsection{Reagents}

Ferrocence ( $\mathrm{Fc}$, supplied by Alderich Chemical Company, Inc.) was employed as the redox probe. Poly(acrylamide) (PAA) (non-cross-linked, average molecular weight: 200,000) was purchased from Alderich Chemical Company, Inc. $1 \mathrm{mg} / \mathrm{mL}$ PAA solution was prepared by adding $10 \mathrm{mg}$ PAA into $10 \mathrm{ml}$ water, then ultrasonicated at $25^{\circ} \mathrm{C}$ until completely dissolved. Tetraethylammonium perchlorate $\left(\mathrm{Et}_{4} \mathrm{NClO}_{4}, \mathrm{TEAP}\right)$ was a polarographic grade product purchased from Nacalai Tesque, Inc. It was dried at $65^{\circ} \mathrm{C}$ for $3 \mathrm{hrs}$ under a high vacuum with $\mathrm{P}_{2} \mathrm{O}_{5}$ before being used. Acetonitrile (AN) was treated with molecular sieves (4A 1/16) dried in vacuum in order to remove water.

\subsection{Preparation of Electrodes}


Gold disk electrodes (ca. $2 \mathrm{~mm} \Phi$ ) were prepared using gold rods housed with Teflon tubes. The gold disks were carefully polished first with emery paper (No.2500), and then with $0.05 \mu \mathrm{m}$ alumina slurry on microcloth pads. After removal of the trace alumina from the surface by rinsing with water, the electrodes were ultrasonicated for $10 \mathrm{~min}$ in fresh piranha solution $\left(\mathrm{H}_{2} \mathrm{SO}_{4}: \mathrm{H}_{2} \mathrm{O}_{2}=3: 1(\mathrm{v} / \mathrm{v})\right)$. Warning: Piranha solution reacts violently with organic solvents. The electrodes were then ultrasonicated using water and anhydrous ethanol. The PAA membrane electrodes were fabricated by transferring a $10 \mu$ d droplet of $0.25,1,2$, or $3 \mathrm{mg} \mathrm{ml}^{-1}$ PAA solution onto the surface of the gold electrodes (respectively designated ME1, ME2, ME3, and ME4), which were then dried in a desiccator for about $4 \mathrm{hrs}$. Geometric area for all electrodes was $3.14 \times 10^{-2} \mathrm{~cm}^{2}$.

\subsection{Electrochemical Measurement}

Cyclic voltammetric and chronoamperometric experiments were performed using a BAS100 Potentiostat CV-50W in a conventional three-electrode system, i.e., a glass cell containing a PAA membrane working electrode, $\mathrm{Ag} / \mathrm{AgNO}_{3}\left(\left[\mathrm{Ag}^{+}\right]=10 \mathrm{mM}\right) / 10 \mathrm{mM} \mathrm{Et} \mathrm{NClO}_{4}$ (for $\mathrm{AN}$ solution) reference electrodes, and a platinum wire counter electrode. The thickness of PAA membrane was determined by optical microscope (Olympus BX41). Cyclic voltammetry at the bare Au electrode was used to confirm the voltammetric characteristics of Fc. A diffusion coefficient of $3.51 \times 10^{-5} \mathrm{~cm}^{2}$ $\mathrm{s}^{-1}$ was calculated from the equation (1) to the peak currents obtained at various voltage scan rates.

$I_{\mathrm{p}}=0.4463\left(n^{3} F^{3} / R T\right)^{1 / 2} D^{1 / 2} V^{1 / 2} c A$

where $I_{\mathrm{p}}(\mathrm{A})$ is peak current, $n$ is the number of electrons transferred per molecule electrolyzed, $F$ represents Faraday's constant, $D$ is the diffusion coefficient of the analyte, $v$ is scan rate, $c$ is bulk concentration of solution, and $A\left(\mathrm{~cm}^{2}\right)$ is electrode area.

\section{Results and Discussion}

\subsection{Double-layer capacitances}

Potential step experiments were performed using the PAA membrane electrode and bare $\mathrm{Au}$ electrode for solution containing only supporting electrolyte (0.1 M TEAP in AN). The applied potential was stepped from $0 \mathrm{mV} v$ s. $\mathrm{Ag} / \mathrm{AgNO}_{3}$ to $200 \mathrm{mV}$. The experimental plot is shown in Fig. 1, from which the double-layer capacitances of the modified electrode and bare Au electrode were obtained. 


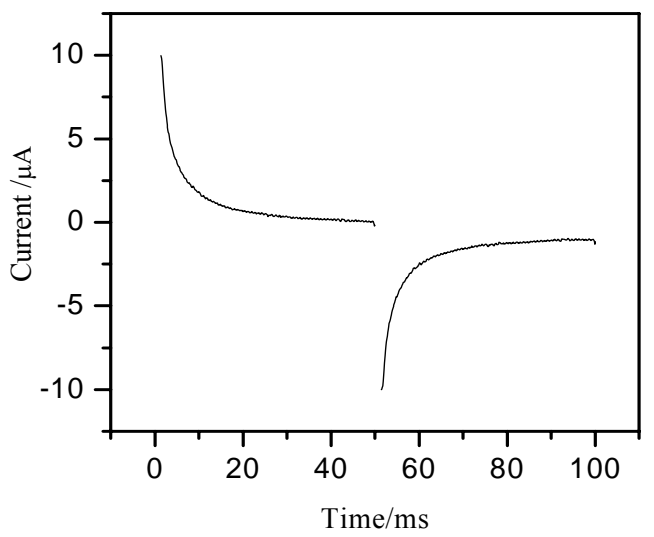

Fig. 1. Experimental chronoamperogram obtained using ME1 for solution containing only supporting electrolyte (0.1 M TEAP in AN).

From the potential step experiment, linear plots of logarithmic current vs. time for the PAA membrane electrodes and bare Au electrode were obtained, as shown in Fig. 2.

The double-layer capacitances of the PAA membrane electrode and bare Au electrode were calculated using the following equations:

$$
\log (i)=\log (u / R s)-t / 2.303 R s C s
$$

$C d=C s / A$

where $i(\mathrm{~A})$ is capacitive current, $u(\mathrm{~V})$ is pulse potential amplitude, $R s(\Omega)$ and $C s(\mathrm{~F})$ are solution resistance and double-layer capacity, respectively, and $C d\left(\mathrm{~F} \mathrm{~cm}^{-2}\right)$ is the double-layer capacitance per square centimeter.

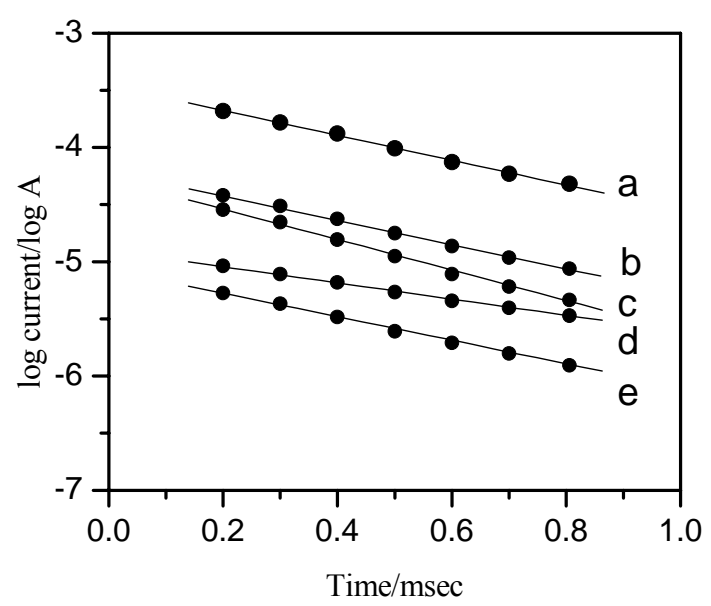

Fig. 2. The logarithmic current response vs. time plot obtained after applying a potentiostatic step of 200 $\mathrm{mV}$ for solution only containing 0.1 M TEAP supporting electrolyte in AN. (a) Bare Au electrode; (b) ME1; (c) ME2; (d) ME3; (e) ME4. 
Combining the slopes and interceptions of the lines in Fig. 2 gave double-layer capacitances of 21, 4.0, 2.8, 1.2, and $0.6 \mu \mathrm{F} \mathrm{cm}{ }^{-2}$ and Rs of $4.0 \times 10^{2}, 3.0 \times 10^{3}, 3.7 \times 10^{3}, 1.6 \times 10^{4}$, and $2.3 \times 10^{4} \Omega$ for bare Au electrode, ME1, ME2, ME3, and ME4, respectively. It is not surprising that the Rs values are different in the same solution because the resistance exists in the PAA pore and this resistance increased with the thickness of the PAA membranes.

Since capacitance current is proportional to electrode active area, the active area of PAA membrane electrode can be obtained from the capacitance. The capacitance of ME1 $\left.(4.0 \mu \mathrm{F} \mathrm{cm})^{-2}\right)$ is about five times less than that of bare Au electrode $(21 \mu \mathrm{F} \mathrm{cm})^{-2}$, signifying that about $19 \%$ of the surface area of Au electrode was active surface. For ME2, ME3, and ME4, the active surface areas were $13 \%, 6 \%$, and $3 \%$, respectively.

\subsection{Voltammetric Response}

To confirm the function of the polymer membrane electrodes, cyclic voltammetry measurements at different scan rates were carried out using $\mathrm{Fc}$ as a redox-active probe in $\mathrm{AN}$.

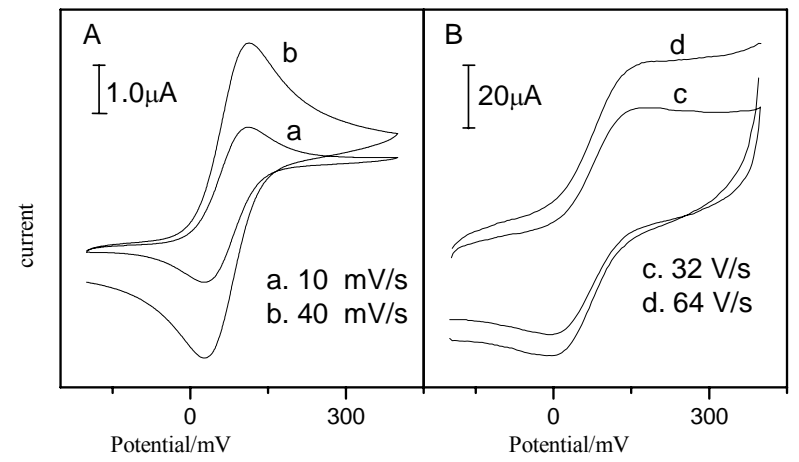

Fig. 3. Cyclic voltammograms of ME1 for $0.5 \mathrm{mM} \mathrm{Fc}$ containing $0.5 \mathrm{M}$ TEAP supporting electrolyte in $\mathrm{AN}$ at various scan rates $\left(v s . \mathrm{Ag}^{+} / \mathrm{Ag}\right)$.

Fig. 3 shows cyclic voltammograms for ME1 at various scan rates. The PAA membrane electrode voltammograms are peak-shaped at low scan rates (Fig. 3 A) and the peak currents are similar in magnitude to the macroelectrode voltammograms ( $I_{\mathrm{p}}$ of bare Au electrode and ME1 were $2.5 \mu \mathrm{A}$ and $2.4 \mu \mathrm{A}$, respectively, at $10 \mathrm{mV} \mathrm{s}^{-1}$ ), which indicated that the total overlap case of radial diffusion had been achieved at these scan rates (vide supra and Fig. 7). The area of the bare Au electrode, $A_{\text {tot }}$, was $3.14 \times 10^{-2} \mathrm{~cm}^{2}$, and the total active area of the PAA membrane electrode, $A_{\text {mem }}$, was $6.46 \times 10^{-3} \mathrm{~cm}^{2}$, on the basis of the double capacitance (19\% active area).

As the scan rate increased, the peak-shape gradually evolved to become sigmoidal-shaped. If the 
scan rate was more than $32 \mathrm{~V} \mathrm{~s}^{-1}$, sigmoidal-shaped voltammograms were observed (Fig. $3 \mathrm{~B}$ ), which shown that radial diffusion of individual electrode occurred in every element at high scan rates (vide supra and Fig. 7).

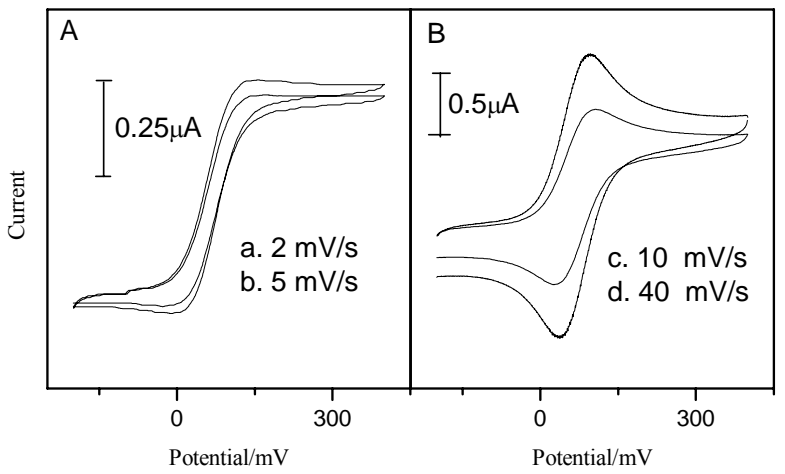

Fig. 4. Cyclic voltammograms of ME2 for $0.5 \mathrm{mM} \mathrm{Fc}$ containing 0.5 M TEAP supporting electrolyte in $\mathrm{AN}$ at various scan rates $\left(v s . \mathrm{Ag}^{+} / \mathrm{Ag}\right)$.

Fig. 4 shows cyclic voltammograms obtained for ME2 at different scan rates. In contrast to the voltammograms observed for ME1, a quasi-steady-state voltammetric response, sigmoidal-shaped voltammograms were observed at low scan rates. These evolved into peak-shaped voltammograms as scan rate increased, the converse of the situation encountered with ME1 (Fig. 3). The limiting current value measured from the oxidative scan was $0.48 \mu \mathrm{A}$ (at $5 \mathrm{mV} \mathrm{s}^{-1}$ ) for ME2. The total active area of the PAA membrane electrode, $A_{\text {mem }}$, was $4.08 \times 10^{-3} \mathrm{~cm}^{2}$, on the basis of the double capacitance ( $13 \%$ active area).

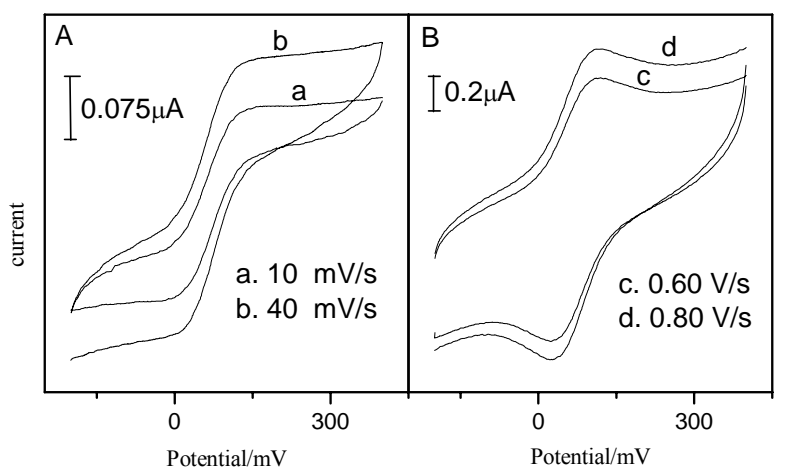

Fig. 5. Cyclic voltammograms of ME3 for $0.5 \mathrm{mM} \mathrm{Fc}$ containing $0.5 \mathrm{M}$ TEAP supporting electrolyte in $\mathrm{AN}$ at various scan rates (vs. $\left.\mathrm{Ag}^{+} / \mathrm{Ag}\right)$.

Fig. 5 shows cyclic voltammograms obtained for ME3 at different scan rates. As with ME2, sigmoidal-shaped voltammograms were observed at low scan rates. The limiting current value 
measured from the oxidative scan was $0.16 \mu \mathrm{A}\left(5 \mathrm{mV} \mathrm{s}^{-1}\right)$. The area of the bare Au electrode, $A_{\text {tot }}$, was $3.14 \times 10^{-2} \mathrm{~cm}^{2}$, and the total area of the PAA membrane electrode, exposed by PAA, $A_{\text {mem }}$, was $1.88 \times 10^{-3} \mathrm{~cm}^{2}$, on the basis of the double capacitance ( $6 \%$ active area). As scan rate increased, the voltammograms evolved from a sigmoidal-shape to a peak-shape.

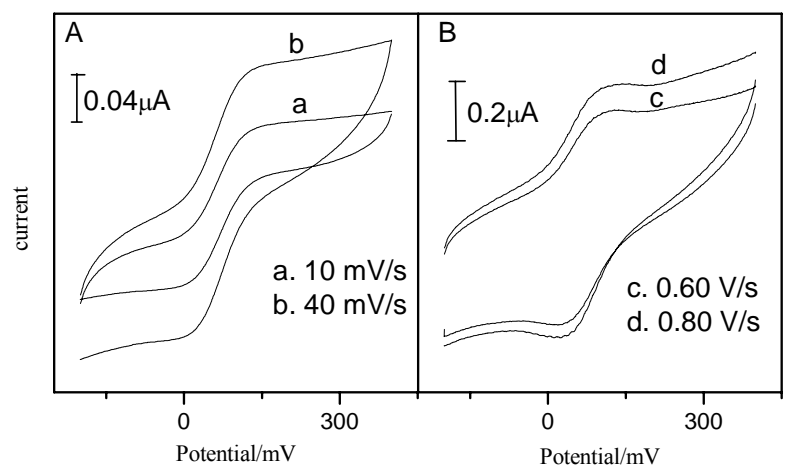

Fig. 6. Cyclic voltammograms of ME4 for $0.5 \mathrm{mM} \mathrm{Fc}$ containing $0.5 \mathrm{M}$ TEAP supporting electrolyte in $\mathrm{AN}$ at various scan rates (vs. $\left.\mathrm{Ag}^{+} / \mathrm{Ag}\right)$.

In the case of ME4, as with ME2 and ME3, there was a quasi-steady-state voltammetric response. Sigmoidal-shaped voltammograms, as shown in Fig. 6, were observed at low scan rates, and these evolved into peak-shaped voltammograms at higher scan rates. The limiting current value measured from the oxidative scan was $0.076 \mu \mathrm{A}$ (at $5 \mathrm{mV} \mathrm{s}^{-1}$ ) for ME4. This indicates that as the thickness of PAA membrane increased, the limiting current value gradually decreased. The total active areas of the PAA membrane electrode, $A_{\text {mem }}$, were $9.42 \times 10^{-4} \mathrm{~cm}^{2}$ for ME4, on the basis of the double capacitance ( $3 \%$ active area).

For these four membrane electrodes, at low scan rates, as thickness of PAA membrane increased, the voltammograms evolved from being peak-shaped to sigmoidal-shaped and peak current decreased. On the contrary, at high scan rates the voltammograms evolved from being peak-shaped to sigmoidal-shaped and the maximum current in the voltammograms decreased gradually.

The results obtained from cyclic voltammetric experiments demonstrated that PAA membrane electrodes possess the properties of microelectrode ensembles when the membrane is not very thick. According to previous investigations [15-18], the voltammetric response at arrays or ensembles of nanoelectrodes or microelectrodes depends on the radii of the elements, the distances between adjacent elements, and the time scale of the experiment. The shape of the voltammogram is dominated by the relative thickness of the radial and linear diffusion zones, which depends on the scan rates and the thickness of the membrane covering the surface of the electrode. 


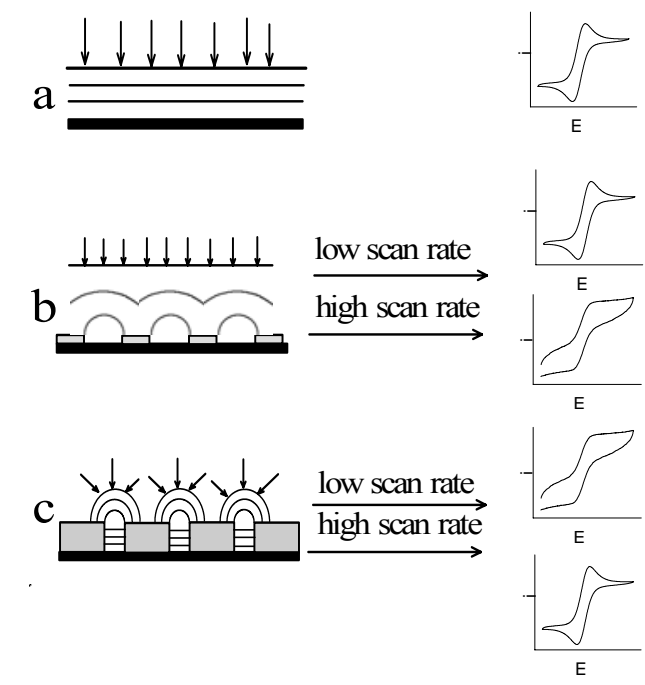

Fig. 7. Schematic illustration showing the diffusion pattern for electrodes for different thicknesses of the PAA membrane. a. linear diffusion to planar electrode; b. thin PAA membrane electrode; c. thick PAA membrane electrode.

For thin membrane electrode (Fig 7 b) at very low scan rates the diffusion layers totally overlap and yield a net linear diffusion field, indicating that the PAA membrane electrode behaves as a planar microelectrode (Fig 7a), and the voltammogram is peak-shaped. In this case, current is proportional to the total geometric area of the electrode. At high scan rates, where radial diffusion fields develop at every element, no overlap is achieved, and the voltammogram becomes sigmoidal. For thick membrane electrode (Fig 7c), where radial diffusion fields develop at every element at very low scan rates, the diffusion layers does not overlap, and voltammogram is sigmoidal. At high scan rates, where the diffusion layers are thin and extend linearly from the individual ensembles elements, a normal peak-shaped, linear diffusion voltammogram should be observed; in this case, currents should be proportional to the electrode active area [17,19].

The experimental results we obtained from cyclic voltammetry are in agreement with the above microelectrode electrochemical theory. In order to confirm the function of PAA membrane electrode as MEEs further, quantitative and semiquantitative analyses of the electrochemical response characteristics of these membranes were also conducted in this study.

\subsection{Semiquantitative Analyses of Voltammetric Data}

Evaluations of voltammetric data were conducted by plotting $\log \left(i_{\mathrm{pa}}\right)$ versus $\log (v)$, where $i_{\mathrm{pa}}$ was the anodic peak current in a Fc voltammogram. Fig. 8 compares plots of $\log \left(i_{\mathrm{pa}}\right) v s . \log (v)$ for ME1, ME2, ME3, and ME4 with a bare Au electrode. 

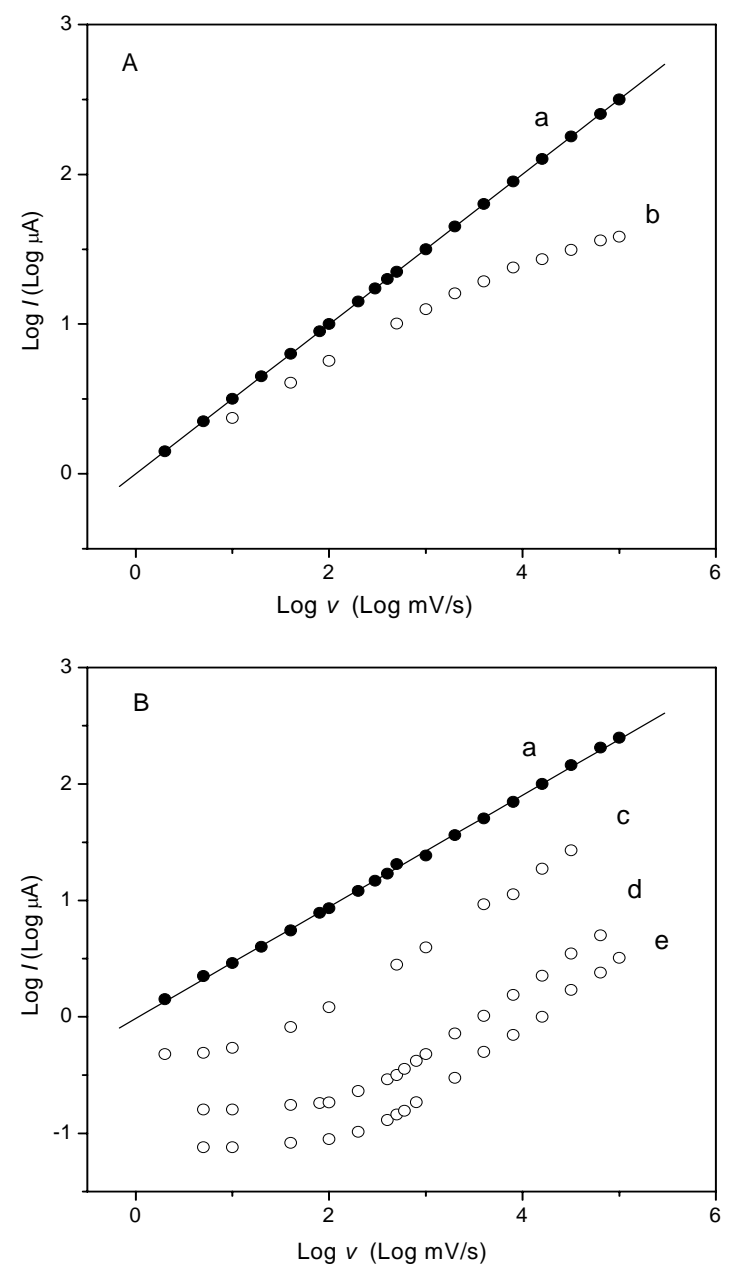

Fig. 8. Log (anodic peak current, $i_{\mathrm{pa}}$ ) vs. $\log$ (scan rate, $v$ ) for cyclic voltammograms of $0.5 \mathrm{mM} \mathrm{Fc}$ containing 0.5 M TEAP supporting electrolyte in AN. (a) bare Au electrode $\left(A=3.14 \times 10^{-2} \mathrm{~cm}^{2}\right)$, (b) ME1, (c) ME2, (d) ME3, (e) ME4.

For the bare Au electrode system, a slope of 0.49 was obtained at all scan rates in our experiment (correlation coefficient, 0.9995), which agreed closely with the theoretical value of 0.5 [20], associated with linear diffusion to the electrode. For the PAA membrane electrode, at low scan rates, where radial diffusion overlapped completely, the slope should be 0.5. ME1 curve (shown in Fig. $8 \mathrm{~A})$ reached the bare Au electrode line, which indicates that the total overlap case had been achieved at low scan rates. With increasing scan rate potential, the slope gradually decreases, and nearly approaches to zero, indicating that radial diffusion became more dominant but also that pure radial diffusion did not occur.

However, as indicated by the voltammograms in Fig. 8B, the slopes obtained for ME2, ME3, and ME4 approach to zero at low scan rates. These responses suggest that pure radial diffusion occurred 
at these three PAA membrane electrodes, the converse of the situation encountered with ME1.

With increasing potential scan rate, all of these three slopes gradually increase. At high scan rates the plots of $\log \left(i_{\mathrm{pa}}\right) v s . \log (v)$ for these PAA membrane electrodes become linear, and the slopes of these lines reach 0.5, indicating that the linear active case has been achieved, as shown in Fig. 8B [14]. At this point, every element (microelectrode) in the PAA membrane was completely independent and again provided peak-shaped voltammograms, which agreed well with the previous investigation [14].

\subsection{Quantitative Analysis}

Chronoamperometry was also used to investigate the voltammetric behavior of the MEEs. Potential step experiments were performed using PAA membrane electrode in $0.5 \mathrm{mM} \mathrm{Fc}$ solution containing 0.1 M TEAP supporting electrolyte in AN. The applied potential was stepped from a value where no faradaic current flowed $\left(-200 \mathrm{mV}\right.$ vs. $\left.\mathrm{Ag} / \mathrm{AgNO}_{3}\right)$ to an applied potential corresponding to the transport limited oxidation of the $\mathrm{Fc}\left(400 \mathrm{mV} v \mathrm{~s} . \mathrm{Ag} / \mathrm{AgNO}_{3}\right)$ during the first step. The potential was stepped back to $-200 \mathrm{mV}$ vs. $\mathrm{Ag} / \mathrm{AgNO}_{3}$ ) for the second part of the chronoamperogram. A typical experimental plot is shown in Fig. 9.

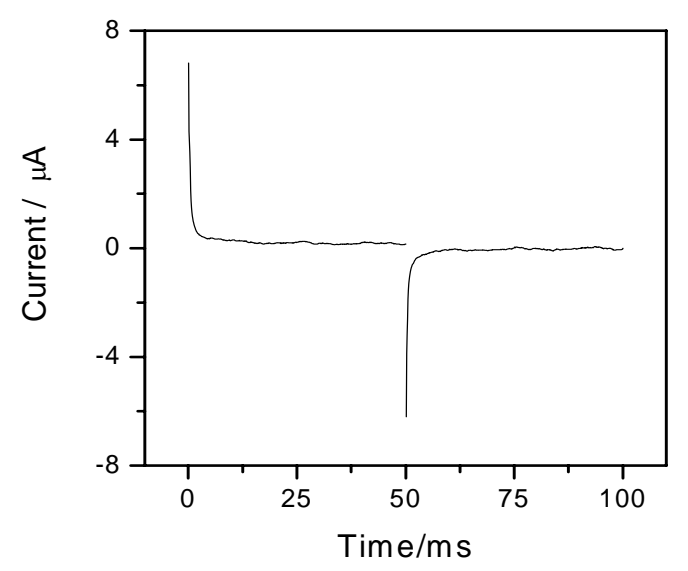

Fig. 9 Chronoamperograms obtained using ME4 for $0.5 \mathrm{mM} \mathrm{Fc}$ solution containing $0.1 \mathrm{M}$ TEAP in AN. The first potential step (from $-200 \mathrm{mV}$ to $400 \mathrm{mV}$ ) was applied at a time, t, of $0 \mathrm{~s}$ and held at $400 \mathrm{mV}$ for $50 \mathrm{~ms}$, then the potential was returned to its initial value, $-200 \mathrm{mV}$, and the current monitored for a further $50 \mathrm{~ms}$.

The plot (Fig. 10) was obtained in terms of the Cottrell equation, which describes the chronoamperometric response where a purely linear diffusion case exists: [18] 
$I=n F A c \sqrt{\frac{D}{\pi t}}$

where $I$ is diffusion current and $t$ is the time of electrolysis.

The plots of $I$ vs. $t^{-1 / 2}$ shown in Fig. 10 are linear at long times. The Cottrell analysis of the chronoamperometric data from the first potential step applied to ME1 (showed in Fig. 10 A) gives an effective area of $3.09 \times 10^{-2} \mathrm{~cm}^{2}$, which approaches to the $A_{\text {tot }}$ of $3.14 \times 10^{-2} \mathrm{~cm}^{2}$, indicating that complete diffusion layer overlap had occurred. This is consistent with the voltammetric observations described above.

The effective area calculated from the slope of the Cottrell plot (Fig. $10 \mathrm{~B}$, a), excluding the current response at short times, for ME2 is $6.34 \times 10^{-3} \mathrm{~cm}^{2}$, which lies between the $A_{\text {mem }}$ and $A_{\text {tot }}$ for this geometry, indicating that partial overlap of the diffusion layers occurred and that the radial diffusion was dominant in this case at long time scale. This is also consistent with the voltammetric observations described above. 

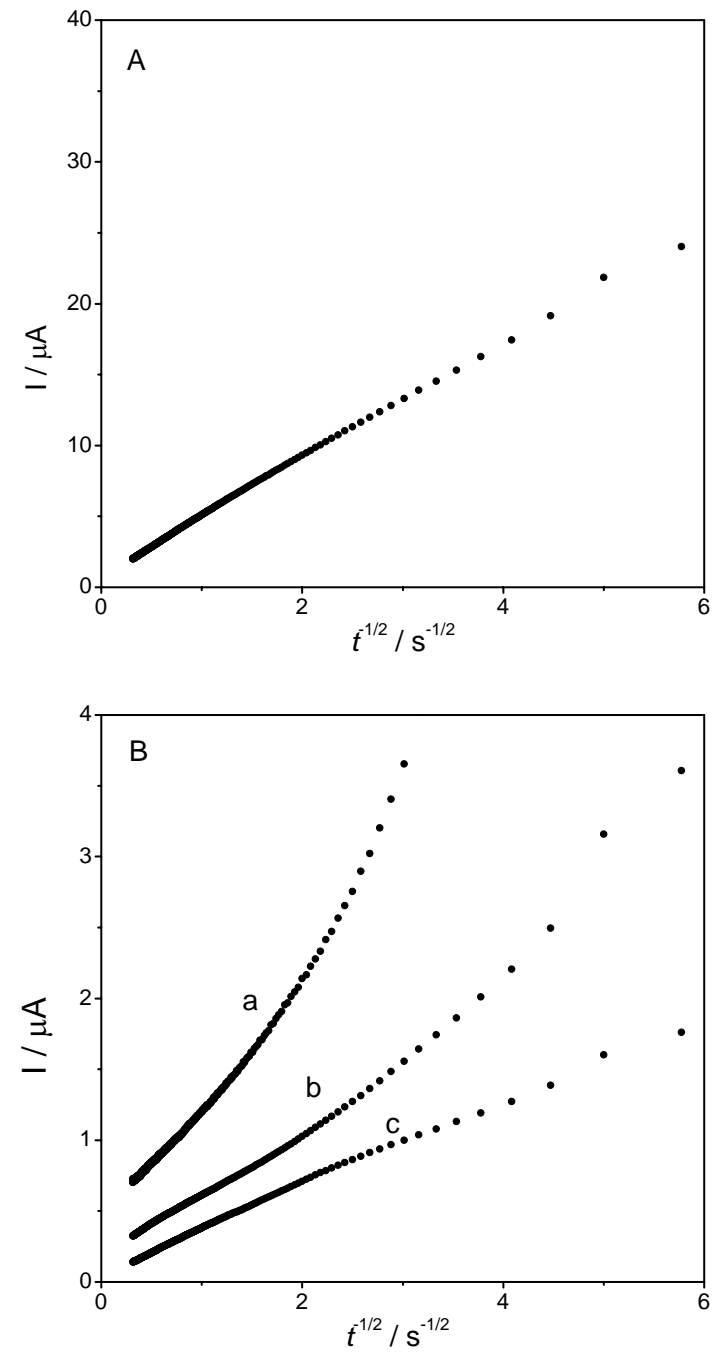

Fig. 10. Cottrell plots (I vs. $t^{-1 / 2}$ ) of the chronoamperometric data obtained for A: ME1, B: a. ME2, b. ME3, and c. ME4.

The Cottrell analysis of the chronoamperometric response obtained from the electrode modified with ME3 and ME4 (as shown in Fig. 10 B, b and c), excluding the current response at short times, gives effective areas of $3.55 \times 10^{-3} \mathrm{~cm}^{2}$ and $2.55 \times 10^{-3} \mathrm{~cm}^{2}$, respectively, which is also intermediate between their $A_{\text {mem }}$ and $A_{\text {tot }}$.

\subsection{Estimation of membrane thickness and pore size}

The PAA membrane thickness estimated by optical microscope is shown in Table 1.

The pore size can be estimated from steady-state current and chronoamperometric data. For an isolated microdisc electrode recessed by a factor $\mathrm{L}$ with respect to its insulating surround, a steady-state limiting current, $I_{\mathrm{lim}}$, is given by the following equation:[21] 
$I_{\lim }=4 n F D c r\left(\frac{\pi r}{\pi r+4 L}\right)$

Where $r$ is the radius of the microdisc, the rest of the variables have the meaning defined for equation (1). Bond et al derived pseudo-analytical expressions for the chronoamperometric response of such a recessed electrode. At short times, the transient response is given by:

$I=n F c r^{2}\left(\sqrt{\frac{\pi D}{t}}\right)\left(1+2 e^{-L^{2} / D t}+2 e^{-4 L^{2} / D t}+\cdots \cdots \cdot\right)$

Combining equation (4) and (5), the following equation can be obtained:

$\frac{I}{I_{\lim }}=\frac{\pi r+4 L}{\pi r}(\sqrt{\pi D})\left(\sqrt{\frac{1}{t}}\right)\left(1+2 e^{-L^{2} / D t}+2 e^{-4 L^{2} / D t}+\cdots \cdots \cdot\right)$

We can see that the plot $\frac{I}{I_{\lim }}$ to $\left(\sqrt{\frac{1}{t}}\right)\left(1+2 e^{-L^{2} / D t}+2 e^{-4 L^{2} / D t}+\cdots \cdots\right)$ should be linear.

Expression (6) is also valid for an array microwell microelectrode if the diffusion field at each micropore electrode does not overlap (short time scale). From the slope of the line, the apparent radius of each micropore could be estimated. The radii of micropores are shown in Table 1, with the exception of ME1, in which no pure steady-state appeared.

Table 1

The thickness and radii of PAA membranes

\begin{tabular}{ccccc}
\hline PAA ME & ME1 & ME2 & ME3 & ME4 \\
\hline$L / \mu \mathrm{m}$ & 0.6 & 2.2 & 4.4 & 6.7 \\
$r / \mu \mathrm{m}$ & - & 6.5 & 11.5 & 18 \\
\hline
\end{tabular}

\section{Conclusions}

The PAA membrane reported here possesses porous properties and can be used to fabricate microelectrode ensembles. Generally, nanoelectrode or microelectrode ensembles are prepared in several ways: (1) template synthesis approach [22,23]; (2) the self-assembled monolayer (SAM) [24,25]; (3) block copolymer self-assembly [16]. Although these approaches are quite effective, they are frequently more complicated. In this study two kinds of ME that had different voltammetric characters were fabricated by a simple method. For one kind of membrane, the voltammograms 
evolved from being peak-shaped to sigmoidal-shaped as scan rates changed from low to high. With the other kind of membrane the converse situation was encountered. The voltammograms evolved from being sigmoidal-shaped to peak-shaped as scan rates increased. The electrochemical responses of the ME fabricated from PAA were in close agreement with the predictions of established microwell electrode electrochemical theory. 


\section{References}

[1] R.L. Solsky, Anal. Chem. 62 (1990) 21R.

[2] E. Bakker, P. Buhlmann, E. Pretsch, Electroanalysis 13 (1999) 11.

[3] J. Bobacka, A. Ivaska, A. Lewenstam, Electroanalysis 15 (2003) 366.

[4] E. Bakker, P. Buhkmann, E. Pretsch, Talanta 62 (2004) 834.

[5] A. Maines, M.I. Prodromidis, S.M. Tzouwara-Karayanni, M.I. Karayannis, D. Ashworth, P. Vadgama, Anal. Chim. Acta 408 (2000) 217.

[6] P.N. Bartlett, P.R. Birkin, Synthetic Metals 61 (1993) 15.

[7] S. Myler, S. Eaton, S.P.J. Higson, Anal. Chim. Acta 357 (1997) 55.

[8] T. Nakamura, K. Makino, M. Ynagisawa, T. Miyasaka, Bull. Chem. Soc. Jpn. 72 (1999) 2459.

[9] T. Nakamura, G.R. Rechnitz, Anal. Chem. 57 (1985) 393

[10] T. Nakamura, J. Ren, T. Hinoue, and K. Umemoto, Anal. Sci., 19 (2003) 991.

[11] T. Miyasaka, Y. Takahashi, and T. Nakamura, Anal. Sci., 17 (2001) 1055.

[12] T. Nakamura, K. Matsuda, M. Takaguchi K. Izutsu, Anal. Sci. 9 (1993) 579.

[13] J. Ren, H. Watanabe, S. Yamamura, T. Nakamura, Anal. Chem. Acta, in press.

[14] I.F. Cheng, , L.D. Whiteley, C.R. Martin, Anal. Chem. 61 (1989) 762.

[15] C. Amatore, J. M. Savéant and D. Tessier, J. Electroanal. Chem. 147 (1983) 39.

[16] E. Jeoung, T.H. Galow, J. Schotter, M. Bal, A. Ursache, M.T. Tuominen, C.M. Stafford, T.P. Russell, V.M. Rotello, Langmuir 17 (2001) 6396.

[17] T. Thurn-Albreht, R. Steiner, J. Derouchey, C.M. Stafford, E. Huang, M. Bal, M. Tuominen, C.J. Hawker, T.P. Russell, Adv. Mater. 12 (2000) 787.

[18] B. Kralj. R.A. W. Dryfe, Phys. Chem. Chem. Phys. 3(2001) 3156.

[19] I.F. Cheng, C.R. Martin, Anal. Chem. 60 (1988) 2163.

[20] R.M. Penner, C.R. Martin, Anal. Chem. 59 (1987) 2625.

[21] A.M. Bond, D. Luscombe, K.B. Oldham, C.G. Zoski, J. Electroanal. Chem. 249 (1988) 1.

[22] Y.D. Jin, S.J. Dong, Communication 1780 (2002).

[23] V.P. Menon, C. R. Martin, Anal. Chem. 67(1995) 1920.

[24] O. Chailapakul, R.M. Crooks, Langmuir 11(1995) 1329.

[25] E. Sabatani, I. Rubinstein, J. Phys. Chem. 91 (1987) 6663. 